\title{
The effect of anxiety on cue selection in the A-Br paradigm '
}

\section{ROBERT L. SOLSO, Loyola University, Chicago, Ill. 60626}

The influence of generalized drive (D) as measured by the Manifest Anxiety Scale (MAS) was examined as it related to paired-associate acquisition, $\mathrm{Br}$ transfer and functional stimulus selection. One-hundred twenty $S s$ were equally distributed through 12 cells of a 2 by 2 by 3 factorial study with the first level being anxiety, the second level being degree of pre-experimental association and the third level being stimulus conditions. Following practice to one perfect trial Ss learned a Br list which corresponded to the respective categories. Modest evidence was found to support the hypothesis that high anxious Ss learn simple paired associates faster than do low anxious Ss but that opposite tendencies were noted in the complex $\mathrm{Br}$ transfer paradigm.

This research paper deals with generalized drive (D) as measured by the Taylor Manifest Anxiety Scale (MAS) and its influence on functional stimulus selection and $\mathrm{Br}$ transfer.

Since Taylor developed the MAS, few concepts in drive theory have excited greater research effort and resulted in more ambiguous findings. The basic rationale of the early studies (Spence, Farber, \& McFann, 1956; Spence, Taylor, \& Ketchel, 1956; Taylor \& Chapman, 1955) was that drive as measured by the MAS facilitates acquisition of simple associates but that as the complexity of the task increases and the possibility of competing responses is enhanced, then high drive impedes acquisition tendencies.

About the same time as the early MAS studies, Easterbrook (1959) suggested that drive level is inversely related to cue utilization: some support for this position is beginning to accumulate (cf. Zaffy \& Bruning, 1966).

In the present study acquisition rates of high and low anxious Ss varying as a function of the task and the theory of cue utilization as a function of drive are examined.

Experimental Design

The overall design was an A-B, A-Br transfer paradigm within a 2 by 2 by 3 factorial arrangement. The factors were: (a) two levels of anxiety, high anxiety (HA) and low anxiety (LA); (b) two acquisition conditions, A-B and A-C; and (c) three stimulus conditions, compound stimulus (A1/A2), simple stimulus color (A1), and simple stimulus verbal element (A2). After Ss attained one perfect trial on first list practice they were asked to relearn another list in which the Rs were repaired with the St of the first list (an $\mathrm{A}-\mathrm{Br}$ list). For purposes of clarity most paradigmatic notations are relative to colors as stimuli and verbal associates to colors as responses. For example, in the A-B acquisition condition, B is a highly frequent verbal response to a color (blue-sky) while in the A-C acquisition paradigm the color may be blue but the verbal response is a word of approximately equal frequency to the $B$ response (blue-hat).

Subjects

The Ss were selected from a pool of 214 students enrolled in a general psychology course who volunteered to complete the MAS. This scale was called "Biographic Inventory" and contained 40 buffer items in addition to the 50 items of the test. Ss were dichotomized into HA and LA groups, with the HA group being composed of Ss whose MAS scores were in the upper $30 \%$ and the LA group being composed of Ss in the lower $30 \%$ of the MAS scores. The mean MAS score for the HA group was 28.00 ; the LA group was 8.55. Ss designated HA and LA were asked to participate in the main experiment and given nominal course credit for their participation. One-hundred twenty Ss (60 HA and 60 LA) were equally distributed throughout the 12 cells of the factorial categories, with $10 \mathrm{Ss}$ in each cell and each $\mathrm{S}$ remaining in the same cellular classification for $\mathrm{Br}$ transfer. Ss were alternately assigned to an experimental condition upon appearance in the laboratory.

Materials

A description of the modified memory drum and lists are identified by Solso (in press). St items were either color patches $6 / 8$ in. $x 7 / 8$ in. (AI terms) or high frequency three-letter words (A2 terms). The compound St conditions (A1/A2) were composed of color rectangles 6/8 in. $x 7 / 8$ in. which contained a verbal element typed (pica type) on a white paper $2 / 8$ in. $x$ 3/8 in. and pasted in the center of the color chip. Thus, the color part of the St comprised an equally distributed $1 / 4$ in. frame around the trigram. $R$ items were either preexperimentally determined high frequency verbal responses to colors (B terms) or verbal items of approx imately equal word frequency count as the B term (C terms) and yet were not associates to colors as measured by Solso (1967).

Eight pairs and three orders were used with $2: 2 \mathrm{sec}$ response anticipation learning and a 4 sec intertrial interval for both original learning and transfer learning.

\section{RESULTS}

The data (cf. Table 1) were treated with two 2 by 2 by 3 factorial analyses: one for $\mathrm{OL}$ and another for $\mathrm{Br}$ transfer.

Original Learning

The first level factor corresponded to HA and LA, the second level to A-B and A-C paradigms, and the third level to compound A1/A2, simple A1, and simple A2 stimuli conditions. Although the factor of anxiety barely reached statistical significance $[F(1,108)=4.21, p<.05]$, the mean trials to criterion was lower for HA Ss in six of the six conditions, in overall acquisition, and in each of the two paradigms ( $A-B$ and $A-C, c f$. Table 2).

The second factor of paradigmatic variation was highly significant with $F(1,108)=20.12, p<.001$, and the third factor of stimulus condition was also highly significant with $F(2,108)=$ $38.97, p<.001$. The Paradigmatic Variation by Stimulus Condition interaction reached significance with $F(2,108)=17.5$, $p<.001$. The three-factor interaction approached significance with $\mathrm{F}(2,108)=2.72$.

Br Transfer

The results of the 2 by 2 by 3 factorial analysis of the $\mathrm{Br}$ transfer were as follows. The factor of anxiety on $\mathrm{Br}$ transfer was close to signilizance with $\mathrm{F}(1,108)=3.75 ;(\mathrm{F}=3.96, \mathrm{p}<.05)$. The second and third main factors were significant with $F(1,108)=4.69, p<.05$ and $F(2,108)=7.03, p<.01$, respectively. Anxiety and paradigmatic variations yielded a significant interaction with $\mathrm{F}(1,108)=7.43, \mathrm{p}<.01$.

Although only modest evidence was found for the deleterious effects of anxiety on $\mathrm{Br}$ transfer, this data becomes more significant when compared with OL. Specifically, within the A-B

Table 1

Mean Trials for Original Learning and Br Transfer

\begin{tabular}{lccccc}
\hline & \multicolumn{2}{c}{ Original Learning } & \multicolumn{2}{c}{ Br Transfer } \\
\cline { 2 - 3 } Paradigm & High Anxiety & Low Anxiety & High Anxiety & Low Anxiety \\
\hline A1-B & 2.8 & 4.0 & 18.4 & 14.1 \\
A2 & & & & 14.1 \\
A1-B & 2.2 & 3.0 & 10.7 & 13.7 \\
A2-B & 14.4 & 18.2 & 20.0 & 12.2 \\
A1 -C & 11.3 & 12.7 & 10.6 & 10.1 \\
A2 & 9.0 & 9.1 & 12.3 & 14.1 \\
A1-C & 13.5 & 14.9 & 18.3 & 13.1 \\
A2-C & & 10.3 & 15.1 & \\
Overall & & & &
\end{tabular}

Table 2

Mean Trials for A-B and A-C Paradigms

\begin{tabular}{lccccc}
\hline & \multicolumn{2}{c}{ Original Learning } & & \multicolumn{2}{c}{ Br Transfer } \\
\cline { 2 - 3 } \cline { 5 - 6 } Paradigm & High Anxiety & Low Anxiety & & High Anxiety & Low Anxiety \\
\hline A-B & 6.5 & 8.4 & & 16.4 & 13.3 \\
A-C & 11.9 & 12.2 & & 13.7 & 12.1 \\
\hline
\end{tabular}




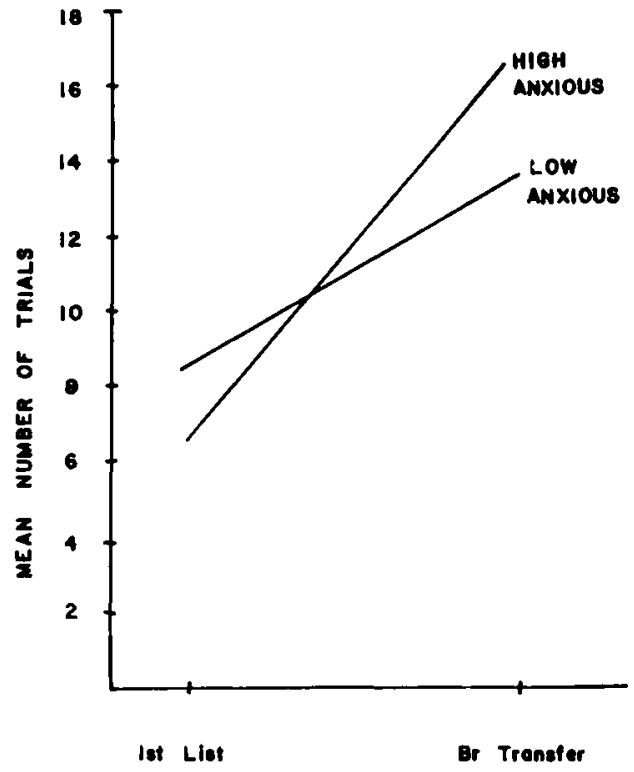

Fig. 1. Mean trials to one perfect trial for $\mathrm{Ss}$ in the A-B, A-Br paradigm.

paradigmatic variation, although one notes the facilitation of acculusition of HA Ss when compared with LA Ss, in the Br transfer the reverse is noted (cf. Fig. 1). This crossover effect, that is, fast acquisition of $\mathrm{OL}$ and then slow acquisition of a $\mathrm{Br}$ transfer list among the HA groups as compared with LA groups, is apparent in four of the six groups, with none of the groups completely reversing the direction.

Finally, OL with $\mathrm{HA}$ and LA groups combined was faster for the A-B paradigm than for the A I $/ A 2-B$ paradigm $(p<.05)$ and faster for Al-C than for the Al/A2-C paradigm $(p<.01)$. Compound stimuli seem to retard acquisition when compared with one component of the compound. Such a finding is important in a stimulus competition hy pothesis.

\section{DISCUSSION}

Modest supporting evidence for the hypothesis of high anxiety facilitating simple paired-associate acquisition is presented in this paper. However, in light of the crossover effect noted in the $\mathrm{Br}$ transfer paradigm the influence of anxiety becomes more significant. If highly anxious $S$ as measured by the MAS are slightly better learners then one may assume that their ability to learn a new list would also be somewhat facilitated. In this paper quite the opposite tendency was found and although the level of significance was low the direction of the relationship was consistent for a variety of paradigms.

A secondary finding of this paper dealt with the stimulus competition hypothesis (Solso, in press). In this hypothesis acquisition of paired associates in which compound stimuli are used is slower than acquisition in which components of a compound stimuli are used. The rationale for this hypothesis is based on backward association. In every OL cell for both HA and LA groups in which compound stimuli appeared there was a corresponding simple stimulus cell which required fewer trials to criterion. thus tending to validate the stimulus competition hypothesis.
Another factor under investigation was the hypothesized inverse relationship between anxiety and cue utilization. In the A 1/A2-B OL cell, one would anticipate early acquisition of the HA group based on drive theory. But also if HA Ss select the color cue and consistently use it as the functional stimulus, then acquisition should be fast because of the pre-experimental conditioning of the color and B response. Conversely if low anxiety Ss examine more St components, then the opportunity for St competition should be enhanced. The results of this experiment offer slight evidence for this argument. In the A1/A2-B OL cell HA Ss reached criterion earlier than LA Ss $(\mathrm{Xs}=2.8$ and 4.0 ). However, both groups reached criterion very early and the stability of this relationship remains problematic.

As noted earlier, significant differences between paradigmatio variations occurred in the Brtransfer. The specific cells in which the differences occurred were those cells in which color served as either part of the St compound A1/A2 or exclusively as the St (A1). Since the A2 stimulus condition was similar for both paradigms and served as a control, no differences were expected or found. However, the differences may be attributed to the influence of pre-experimental associations to the color terms in the $\mathrm{Al} / \mathrm{A} 2-\mathrm{B}$ and $\mathrm{A} / \mathrm{B}$ conditions. The learning model may be conceptualized as A-B (pre-experimental), $\mathrm{A}-\mathrm{B}, \mathrm{A}-\mathrm{Br}$ as contrasted with $A-B$ (pre-experimental), A-C, or A-Cr. The influence of pre-experimental associations plus laboratory associations (A-B, A-B) may form a stronger association than A-C laboratory association. The presence of this well-formed relationship may manifest itself in increased associative interference in $\mathrm{Br}$ learning. This finding is consistent with Solso (1968) although in the present paper the influence of pre-experimental associates on $\mathrm{Br}$ transfer is more obvious.

Also in the $\mathrm{Br}$ transfer significant main variations were observed in the third factor (stimulus conditions) with the greater disparity between word stimuli and color stimuli. This phenomenon may be attributable to stimulus similarity. Although it is possible to conceptualize colors as having some form of similarity with each other, they also have some very unique and distinguishable characteristics. The degree of stimulus similarity of the verbal elements may be greater than the color similarity.

\section{REFERENCES}

EASTERBROOK, J. A. The effect of emotion on cue utilization and the organization of behavior. Psychological Review, 1959, 66, 183-201.

SOLSO, R. L. Meaningfulness of colors. Paper read at Midwestern Psychological Association, Chicago, Illinois, May, 1967.

SOLSO, R. L. Word associates to color and the $\mathrm{Br}$ paradigm. Psychological Reports, 1968, 22, 494.

SOLSO, R. L. Functional stimulus selection as related to color versus verbal stimuli. Journal of Experimental Psychology, in press.

SPENCE, K. W., FARBER, J, E., \& McFANN, H. H. The relationship of anxiety (drive) level to performance in competitional and noncompetitional paired-associates learning. Journal of Experimental Psychology, 1956, 52, 296-305.

SPENCE, K. W., TAYLOR, J., \& KETCHEL, R. Anxiety (drive) level and degree of competition in paired-associates learning. Journal of Experimental Psychology, 1956, 52, 306-310.

TAYLOR, J. A., \& CHAPMAN, J. P. Anxiety and the learning of paired associates. American Journal of Psychology, 1955, 68, 671.

ZAFFY, D. J., \& BRUNING, J. L. Drive and the range of cue utilization. Journal of Experimental Psychology, 1966, 71, 382-384. NOTE

1. The author wishes to express his appreciation to the following individuals who assisted in this experiment: L. Coalwell, J. Fuqua, J. Hanson, J. Johnson, M. Rivinus, and G. Schatz. Parts of this paper were read at the Midwestern Psychological Association Meeting, May, 1968, Chicago. 